\title{
A method to evaluate relevance of hemodynamic factors to artery bifurcation shapes using computational fluid dynamics and genetic algorithms
}

\author{
Masako HIMENO, ,*, Shigeho NODA*, Kazuaki FUKASAKU ${ }^{* * *}$, \\ Ryutaro HIMENO * and Shigeru TADANO** \\ *Advanced Center for Computing and Communication \\ Hirasawa 2-1, Wako, Saitama, 351-0198, Japan \\ ** Graduate School of Engineering, Hokkaido University \\ Kita 8 Nishi 5, Kita-ku, Sapporo, Hokaido,060-0808, Japan \\ E-mail: mhimeno@riken.jp \\ *** Center of Advanced Photonics, RIKEN \\ Hirasawa 2-1, Wako, Saitama, 351-0198, Japan
}

Received: 9 August 2016; Revised: 7 November 2016; Accepted: 12 April 2017

\begin{abstract}
There are many hemodynamic factors which have been suggested to relate to artery shape regulation but it has not been certain which factors are more effective. We propose a new method to evaluate the relevance of hemodynamic factors to artery shape. First, after selecting some associated factors said to be related with artery shapes, we performed multi-objective optimization that sets two of their factors as objectives to obtain optimized shapes using computational fluid dynamics and genetic algorithms. Then, we checked how similar the original shape was to the optimized shapes using Objective Difference Index (ODI). After this process was applied to seven typical carotid artery bifurcation shapes (seven actual cases), the relevance of each combination of two factors was evaluated. We selected five factors: a) to minimize maximum time-averaged Wall Shear Stress (WSS), b) to maximize minimum time-averaged WSS, c) to minimize WSS gradient, d) to minimize WSS temporary gradient, and e) to minimize inner surface area. At the first stage, shapes were optimized by using only radius as the variable using a fixed center line. We set six kinds of combinations of factors that have trade-off relationships. As a result, ODI in the cases of a) and e) was the smallest, having a value was about one-twentieth that of ODI for the second smallest combination factors of c) and e). Combination factors of minimizing both maximum WSS and artery radius were evaluated to be the most relevant to artery radius in the six kinds of tested combinations. At the next stage, by setting both the radius and center line as variables, it became clear that this combination was also related to the position of the center line. We confirmed our method effectively evaluates the relevance of factors to artery bifurcation shapes.
\end{abstract}

Key words: Carotid artery bifurcation, Computational fluid dynamics, Genetic algorithms, Multi-objective optimization, Wall shear stress, Relevance of factors

\section{Introduction}

There have been various studies in artery adaptation and development. Animal studies have been undertaken concerning the straight parts of arteries (Masuda, et al., 1999, Tohda, et al., 1992, Langille and O'Donnell, 1986, Sho, et al., 2004). They reported that high wall shear stress (WSS) caused by an increase of blood flow enlarges blood tube radius. Decrease of blood flow makes WSS lower and radius smaller. However, blood flow at the bifurcation part is significantly different from that at a straight part. Figure 1 compares calculated flows between a straight artery and a carotid artery bifurcation at a diastole. In the straight part of the artery, blood flows almost parallel to the wall, and WSS is mostly constant in the circumferential direction. On the other hand, at the bifurcation, complex flows such as 
reverse flow and swirling arise. Therefore, WSS distribution differs from that of straight areas, and WSS value is not uniform on the same circumference. How WSS regulates artery shape at bifurcation parts could not be easily predicted.

Additionally, endothelial cells were observed to respond to each factor of WSS Gradient (WSSG), WSS Temporary Gradient (WSSTG), and Oscillating Shear Index (OSI) (Bao, et al., 1999, DePaola, et al., 1993, Chiu and Chien, 2011). Those factors were induced by disturbed flow such as reverse flow and swirling. However, whether and how those factors regulate normal artery bifurcation shapes has not been revealed, but they have been thought to relate to the initiation and development of abnormal shapes such as aneurysm and stenosis (Meng, et al., 2007, Chiu and Chien, 2011).

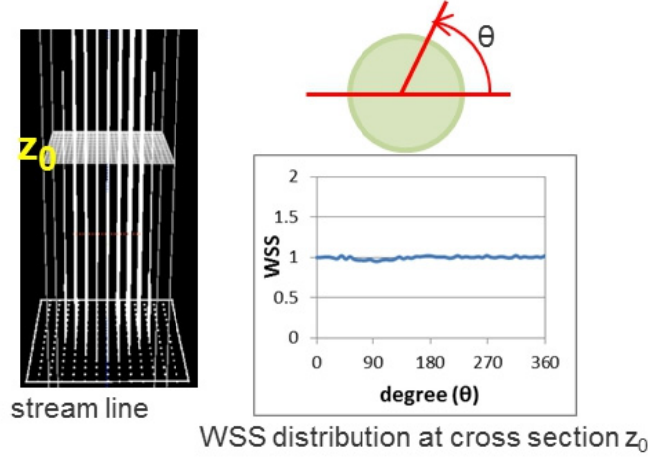

(1) Straight artery

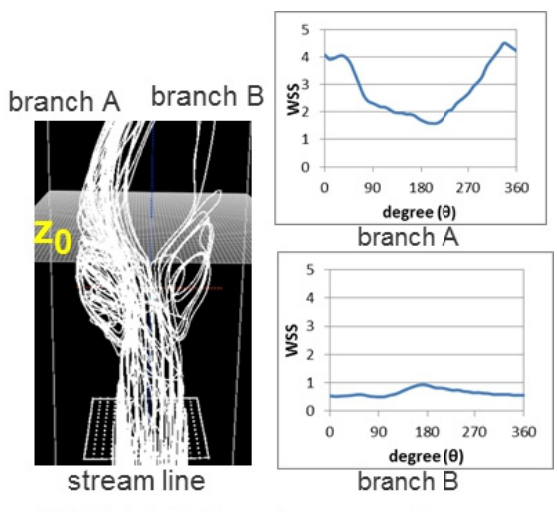

WSS distribution at cross section $\mathrm{z}_{0}$

(2) Carotid artery bifurcation

Fig. 1 Comparison of flow fields between a straight artery (1) and a carotid artery bifurcation (2) at diastole.

We estimate that the identification or effectiveness of hemodynamic factors related to artery shape would be evaluated with computer simulations by solving the optimization problem: shapes would adapt optimally to some kind of hemodynamic factors. An asymptotic method has often been used to obtain an optimized structure or remodeling process to study adaptation mechanisms. Bone structure change under the conditions of various level of stress was obtained with this method (Tsubota et al., 2002, Nowak, 2006). Moreover, orientation of collagen fibers in the blood wall was optimized to investigate their relationship to circumference stress. (Hariton et all, 2007). Those problems generally have a single optimum solution and linear function. On the other hand, there are large variations among individuals at bifurcation parts (Schlz and Rothwell, 2001, O’Flynn et al., 2011). Common factors having all variations might not be independent of each other. Additionally, at bifurcation parts, only a slight variation of geometry often causes to unexpected and large variation of flow (Bijari, et al., 2012). This problem would likely have multiple optimum solutions and a non-linear function. In this study, we applied a Genetic Algorithm (GA) as the optimizing method. GA is a probability-based search method based on the principle of inheritance and evolution in nature (Goldberg, 1989). GA can be used relatively easily to solve a problem having non-linear function and multiple optimum solutions. Various GA have been designed to solve difficult real-world optimization problems (Sasaki, et al., 2004, Takahashi and Kobayashi, 2001, Himeno and Himeno, 2003). Therefore, we used GA to obtain optimized shapes.

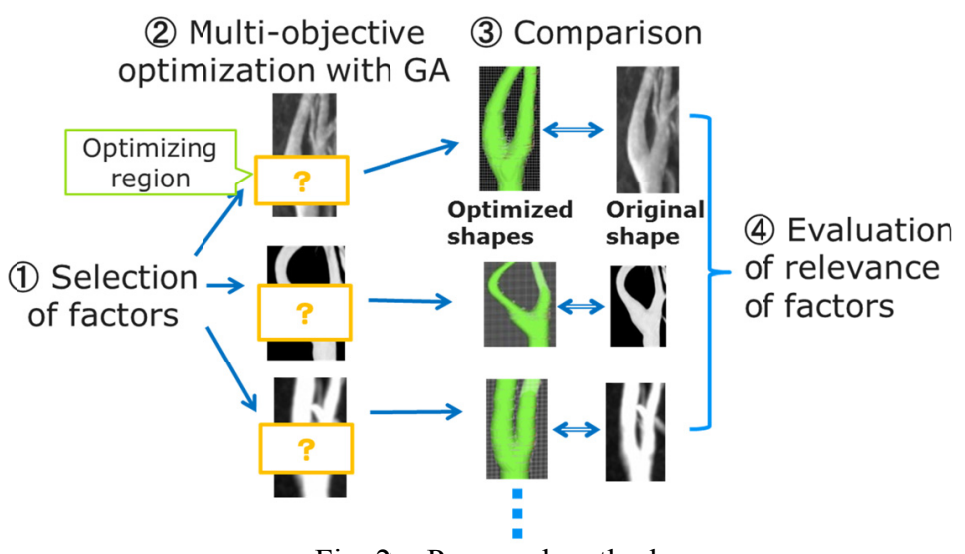

Fig. 2 Proposed method. 
We developed a method to evaluate the relativity of hemodynamic factors to artery bifurcation shapes using GA and computation fluid dynamics (CFD) (shown in Fig. 2). First, we selected factors that might relate to artery shapes. Second, multi-objective optimization of the arterial radius and center line that sets the selected factors as objectives was performed to obtain a series of optimized shapes on the basis of an actual artery shape. For initial variations, we prepared randomly generated shapes, excluding the original shape. Third, we checked how much each optimized shape differed from the original. Applying this process to several different actual cases, we evaluated the relevance of the selected factors.

\section{Methods}

\subsection{Hemodynamic factors in optimal processing}

There are more than ten kinds of proposed hemodynamic factors that are said to affect normal or abnormal artery shape, such as low value or high value of WSS, OSI, WSSG, and WSSTG (Kamiya and Togawa, 1980, Langille and O’Donnell, 1986, Sorop, et al., 2003, Chiu, et al., 2004, Kelly and Snow, 2007, DePaola, et al., 1993, Bao, et al., 1999). We chose five factors: 1) maximum time-averaged WSS (max WSS), 2) minimum time-averaged WSS (min WSS), 3) maximum time-averaged WSSG (max WSSG), 4) maximum time-averaged WSSTG (max WSSTG), and 5) inner surface area. We checked whether our proposed method differentiates the relevance of those five factors or not.

These five factors are explained more in detail hereafter. The first factor: max WSS is chosen because artery radius might be regulated locally so as not to have an excessively high time-averaged WSS (meaning that max WSS is less than a moderate value). The second factor: min WSS is chosen because artery radius is regulated locally so as not to have an excessively low time-averaged WSS. At bifurcations, WSS distribution differs from that of straight areas, and WSS value is not uniform for the same circumference (Fig. 1). It is not known how artery radius is regulated in such cases. We presumed that it is regulated in the circumferential direction so that max WSS for circumference is less than a moderate value in the case of the first factor, because a fibrous structure forms mainly in the circumferential direction within the artery wall (Canham, et al., 1989, Finlay, et al., 1995). In the same way, in the case of the second factor, we presumed the artery radius to be regulated locally so that min WSS for circumference is higher than a certain value. In the same way, we presumed that the artery radius is regulated locally so that max WSSG or WSSTG for circumference is less than a certain value for the third and the fourth factors. These WSS-related factors were calculated as follows. Fist, instantaneous WSS, WSSG, and WSSTG of the surface distribution were calculated in one heartbeat and time-averaged. Then their maximum or minimum value was searched for and selected in the optimizing region (described in 2.2.2). Besides hemodynamic factors, we took a factor of material saving point of view, which is minimizing inner surface area for optimizing.

We assumed that the feedback system would regulate the artery radius so as to keep the blood flow in a moderate condition. Therefore, we performed multi-objective optimization in these six combinations of the objectives, which have trade-off relationships, as the test functions:

F1: minimization of max WSS and minimization of inner surface area

F2: minimization of max WSS and maximization of min WSS

F3: minimization of max WSSG and minimization of inner surface area

F4: minimization of max WSSG and maximization of min WSS

F5: minimization of max WSSTG and minimization of inner surface area

F6: minimization of max WSSTG and maximization of min WSS

\subsection{Targeted bifurcation part: Carotid artery bifurcation}

Carotid artery bifurcations were selected as targets because they widely vary in shape from person to person (Schlz and Rothwell, 2001), which makes it very adequate to study the relevance of each factor.

We selected seven typical artery shapes (A to G shown in Fig. 3) from 13 artery shapes of volunteers whose ages ranged from their 20s to 80s. Those shapes were reconstructed with the images of Magnetic Resonance Angiography (MRA): Philips Achieva 1.5T or Computerized Tomography (CT): Philips Brilliance CT16. The ethical review board of RIKEN approved the experimental protocol, and consent was obtained from all subjects.

There are large variations among individuals in terms of degree of bifurcation angle and size of the carotid bulb, which is an expanded part from Common Carotid Artery (CCA) to Internal Carotid Artery (ICA). The angles between ICA and External Carotid Artery (ECA) of the seven artery shapes we used were from 30.3 to 87.3 degrees, while 
Thomas et al. reported those of a range of 50 shapes were from 31.2 to 97.6 degrees (Thomas, et al., 2005). In terms of the ratio of cross sectional area from inlet to the max value of carotid bulb, which is relevant to the size of the carotid bulb, the ratios of seven artery shapes were from 1.32 to 2.21 . While, Thomas et al. also reported that the ratio was from 1.17 to 2.42 . The seven shapes vary enough because those ranges cover more than $70 \%$ of Thomas et al.'s report ( $86 \%$ of the bifurcation angle and $71 \%$ of the carotid bulb expansion ratio).

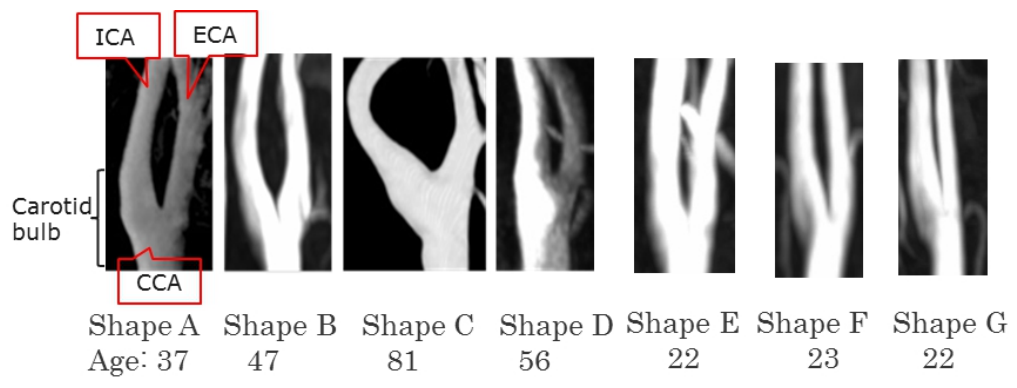

Fig. 3 Tested original shapes of carotid arteries.

\subsection{Optimizing region and pre-fixed region for shape optimization}

Figure 4(1) shows an optimizing region (changeable region) and pre-fixed regions. The top of this figure is the direction of the head, and the bottom is the direction of the heart. In this study, we focused on ICA for optimization, because carotid artery bifurcation is characterized by an expansion of ICA, which varies greatly from individual to individual. Therefore, we only optimized the carotid bulb region. We set a region from just before the increase of the artery radius $\left(S_{\mathrm{st}}\right)$ to just after the decrease in radius $\left(\mathrm{S}_{\text {ter }}\right)$ of ICA as an optimizing region (Fig. 4(1)), which was equivalent to the region of the carotid bulb. The other regions of ICA and ECA are set as the pre-fixed region, in which the original shape was used. To reconstruct the bifurcation shape, ICA and ECA were separately defined. Additionally, CCA was created by merging both branches. Each branch has the parameters of both the series of radii and position of the center line.

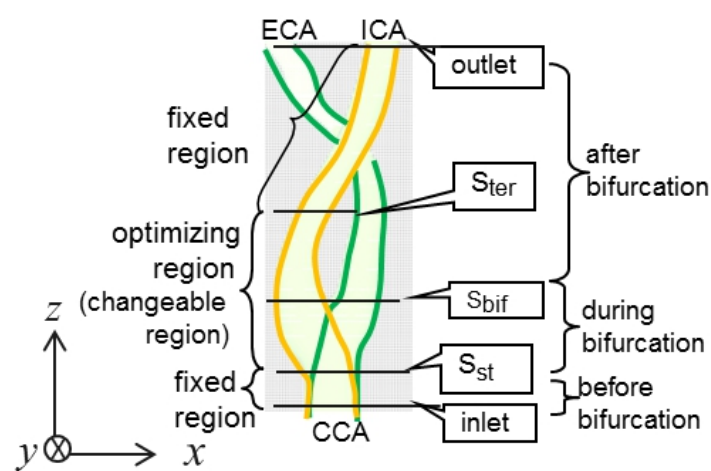

(1) Optimizing region and fixed region

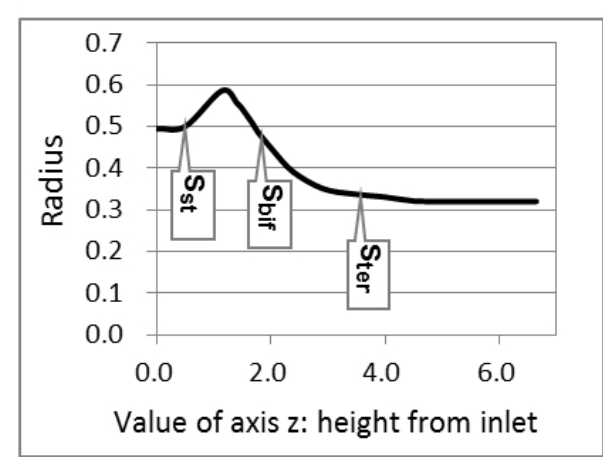

(2) Radius of ICA of shape A

Fig. 4 Illustration of reconstructing artery with bifurcation. (1) The artery is divided into two regions: pre-fixed regions and optimizing region (changeable region). (2) Positions of $\mathrm{S}_{\mathrm{st}}, \mathrm{S}_{\mathrm{bif}}$, and $\mathrm{S}_{\mathrm{ter}}$ are defined from the variations of radii of ICA of original shape A. Radius and value of axis $\mathrm{z}$ were normalized by diameter at inlet.

To define the optimizing region, the variations of ICA of the original shapes were measured. Figure 4(2) displays radii of ICA in the case of shape A. In the figure, the vertical axis is the radius, and horizontal axis is the value of axis $\mathrm{z}$ shown in Fig. 4(1), whose origin is the position at the inlet and whose direction is from heart to head. Values of both axes were normalized by the diameter at the inlet. First, we selected three positions: $\mathrm{S}_{\mathrm{st}}$, the end of the bifurcation ( $\left.\mathrm{S}_{\mathrm{bif}}\right)$, and $S_{\text {ter }}$. We set the difference of $z$ value between the inlet and $S_{s t}$ to the value of CCA radius. The difference between the outlet and $\mathrm{S}_{\text {ter }}$ was set to 1.5 times the difference between $\mathrm{S}_{\text {bif }}$ and $\mathrm{S}_{\text {ter. }}$. How to reconstruct a three-dimensional shape was described in Appendix 1.

\subsection{How to define shape parameters in the optimizing region}

Figure 5(1) shows the method to define ICA radius in the optimizing region. The values of both axis $\mathrm{z}$ (the origin 
was set as the position at the inlet) and radius were normalized with the diameter at the inlet. The radius in the optimizing region was defined using a spline function with six points. Fixed points were the inlet point, $S_{\text {st }}$, $S_{\text {ter }}$, and $\mathrm{S}_{\text {ter+1 }}$. The difference of $\mathrm{z}$ value between $\mathrm{S}_{\text {ter }}$ and $\mathrm{S}_{\text {ter+1 }}$ is a quarter of the difference between the bifurcation point ( $\mathrm{S}_{\mathrm{bif}}$ ) and $\mathrm{S}_{\text {ter }}$. Optimizing points were control points 1 (set between $\mathrm{S}_{\mathrm{st}}$ and $\mathrm{S}_{\text {ter }}$ ) and 2 (set between $\mathrm{S}_{\text {ter }}$ and $\mathrm{S}_{\text {ter }}$ ) in regions 1 and 2, respectively. There were four variables (each control point has two variables: radius (r) and value of axis $\mathrm{z}$ ).

A series of center positions $\mathrm{P}(\mathrm{x}, \mathrm{y})$ was also defined using a spline function in a way similar to that in the radius case. In this case, there were six variables. Each control point (optimizing points) has three variable: three components of position $(\mathrm{x}, \mathrm{y}, \mathrm{z})$. The origin was set at the center of CCA of a cross-sectional two-dimensional plane at the inlet.

Next, seven original shapes were reconstructed using these parameters and checked each value of ranges. Then, the ranges were multiplied by 1.5 to have enough variations and set as follows: (these values were normalized with the diameter at the inlet)

$$
\begin{aligned}
& 0.18 \leq r \leq 0.8 \\
& 0 \leq x, y \leq 2
\end{aligned}
$$

Figure 5(2) shows examples generated on the basis of shape A.

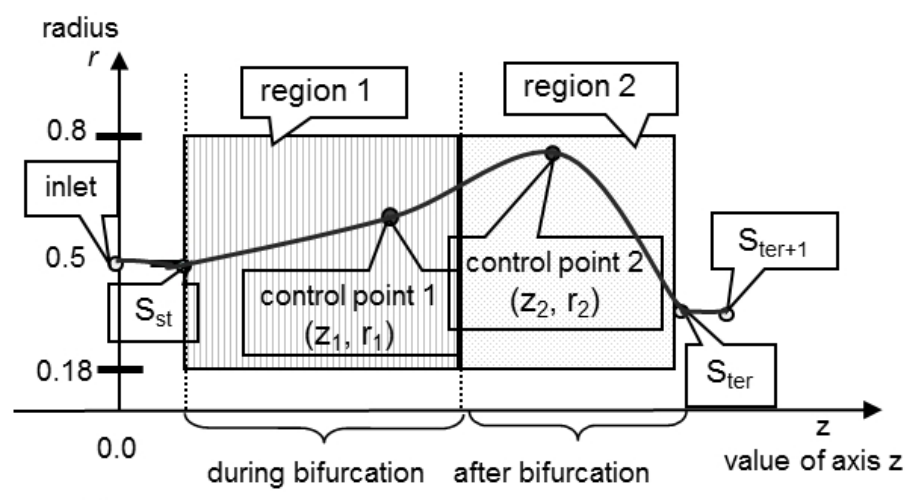

(1) How to define radii in the optimizing region

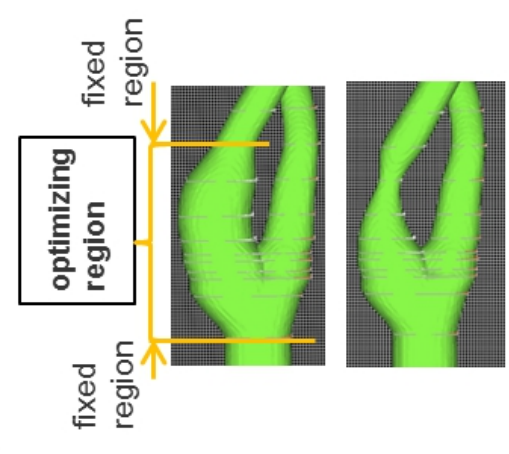

(2) Examples created on the basis of shape $A$

Fig. 5 (1) Each radius along the artery in the optimizing region was defined using a spline function with six points. Fixed points were the inlet, $S_{\mathrm{st}}, \mathrm{S}_{\mathrm{ter}}$, and $\mathrm{S}_{\mathrm{ter}+1}$ (see Fig. 4), while optimizing variables were control points 1 and 2 in regions 1 and 2, respectively. $r$ and $z$ were normalized by diameter at inlet. (2) Examples generated on the basis of shape A with two control points selected from the optimizing area at random.

We took a two-stage approach for optimization. Bijari et al. studied which geometric variables cause disturbed flow at the carotid artery bifurcation and reported that expansion of the proximal to the flow divider, which is the change in artery radius, leads to an increase of surface area having low WSS (Bijari, et al., 2012). Therefore, for the first stage, we performed optimization using only the ICA radius as a variable and using a fixed center line and examined the relationship between artery radius and WSS factors. Next, for the second stage, treating both the radius and center line as variables, we optimized candidate factors on the basis of results from the first stage. The total numbers of variables were four and ten at the first and second stages, respectively.

\subsection{Blood flow analysis}

Blood flow was analyzed using a CFD code we had developed. The schemes used in this code (Noda, et al., 2006) are voxel approach coupled with Volume of Fluid (VOF) and Quadratic Upstream Interpolation for Convective Kinematics (QUICK). The voxel approach usually has more errors than the body fitted approach because it approximates a curved surface as a stairway. Therefore, we coupled the voxel approach with the VOF scheme to overcome this stairway approximation. From the checking errors due to the VOF approach shown in Appendix 2, we decided to use the voxel size of 0.1 .

The maximum Reynolds number based on the radius of the inlet and maximum velocity of pulsatile flow at the inlet is 1,000 (cited from literature (Dammers, et al., 2003, Reneman, et al., 2006)) for the blood flow simulation. We used pulsatile flow with Poiseuille velocity distribution for the inlet flow. Although each velocity at the inlet may differ among shapes A to G, we used the same flow pattern for one heartbeat cited from literature (Poepping, et al., 2002), because Hoi et al. reported that using a subject-specific vs. assumed flow pattern only modestly affects the exposure to 
blood flow (Hoi, et al., 2010).

\subsection{Shape optimization method: Multi-objective genetic algorithm (MOGA)}

Each test function (F1 to F6) has trade-off relationship between objectives. In this case, we do not obtain a single optimized shape but obtain a series of optimized shapes called Pareto-front. We show the outline for multi-objective optimization and for GA to solve multi-objective problems (Multi-Objective Genetic Algorithm: MOGA) in Appendix 3 .

We used Non-dominated Sorting GA (NSGA II) (Deb, et al., 2002), which is generally used for multi-objective optimization, to obtain a series of trade-off shapes. NSGA II is a method that provides trade-off solutions at one time without any weight parameters indicating the interaction between objectives. The procedure of MOGA coupling CFD used in this study is as follows (Fig. 6). 1) A certain number of candidate shapes was generated as an initial population, and WSSs for those shapes were calculated by CFD analysis. For the populations, we took 30 and 40 shapes at the first and second stages, respectively, from the results of a preliminary study. 2) New candidate shapes were generated with a recombination of current shapes using UNDXm (Ono and Kobayashi, 97), which is one of the generally used recombination procedures. 3) CFD analysis was performed on new candidate shapes. 4) Trade-off shapes at that time were selected as the next candidate population: a set of next candidate shapes. By repeating steps 2) to 4), shapes were searched for from multiple points in the search domain. Each repetition of 2) to 4) is called a generation. Searching was performed until the rate of new shapes became less than 0.05 , at which point it was recognized that the population was sufficiently converged (Jaimes and Coello, 2005). GA was performed in three trials with different initial populations for each kind of optimization (42 kinds of optimization: six kinds of test functions $\times$ seven cases described later).

For all calculations, we used a PC cluster, RICC at RIKEN, to reduce computation time by parallelization (Himeno, et al., 2010). It took about two hours to compute one shape with 32 CPUs, and 61 hours in the case of the first stage and 100 hours in the case of the second stage to optimize one GA trial on average.

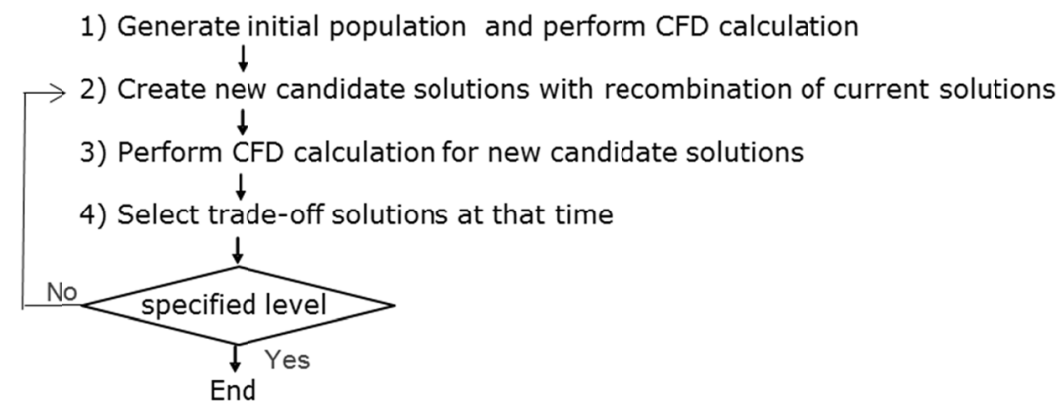

Fig. 6 The procedure of MOGA coupling CFD

\subsection{How to compare optimized shapes and original shape: Objectives Difference Index (ODI)}

To check whether the original shape was included among Pareto-front or not, the similarity between them was needed to measure quantitatively. Sometimes it happens in flow phenomena that a little variation of specific region of shape leads to large difference in flow (Bijari, et al., 2012). Therefore, we measured the degree of difference between them, from the optimized result graph using two objective values as coordinates as shown in Fig. 7 . We call it Objectives Difference Index and the ODI is defined as minimum distance between the original shape's point and the Pareto-front (a series of optimized shapes) curve on the graph.

Figure 7 shows all points of shapes including the points in the optimizing process. Black circles $M_{1}$ to $M_{n}$ are on Pareto-front and triangles show solutions in the optimizing process. $\mathrm{P}$ shows the point of original shape. ODI was defined as follows. First, picking out two neighboring points $\left(\mathrm{M}_{\mathrm{i}}, \mathrm{M}_{\mathrm{i}+1}\right)$ on a graph, we calculated the minimum distance $\left(O D_{i}\right)$ from the original shape to the line connecting $M_{i}$ to $M_{i+1}$. Next, using $n$ points of $M$, we called the minimum OD among $\mathrm{OD}_{1}$ to $\mathrm{OD}_{\mathrm{n}-1}$ "ODI". In this process, each objective value was normalized with the each variation range at all points in the optimizing process (black circles and triangles). Each variation range was set with maximum value $\left(\mathrm{Ob}_{\mathrm{j}_{\_} \max }\right)$ and minimum value $\left(\mathrm{Ob}_{\mathrm{j}_{-} \min }\right)$ in Fig. (7) and Eq. (3). In Eq. 3, $\mathrm{M}_{\mathrm{i}}$, and $\mathrm{P}$ ' were normalized points with 
variation ranges

$$
O b_{j_{-} M^{\prime} i}=\frac{\left(O b_{j_{-} M i}-O b_{j_{-} \min }\right)}{\left(O b_{j_{-} \max }-O b_{j_{-} \min }\right)} \quad, \quad O b_{j_{-} P^{\prime}}=\frac{\left(O b_{j_{-} P}-O b_{j_{-} \min }\right)}{\left(O b_{j_{-} \max }-O b_{j_{-} \min }\right)}
$$

where $j$ indicates each objective and is 1 or 2 as shown in Fig. 8.

This range corresponded to the range of the search domain. ODI was selected among normalized values of OD' ${ }_{i}($ Eq. (4) and (5)).

$$
\begin{aligned}
& O D_{i}^{\prime}=\text { minimum distance }\left(\operatorname{Line}\left(M_{i}^{\prime}, M_{i+1}^{\prime}\right), P^{\prime}\right) \\
& \text { Evaluating Objectives difference index }: O D I=\min \left(O D_{1}^{\prime}, O D_{2}^{\prime}, \cdots O D_{n-1}^{\prime}\right)
\end{aligned}
$$

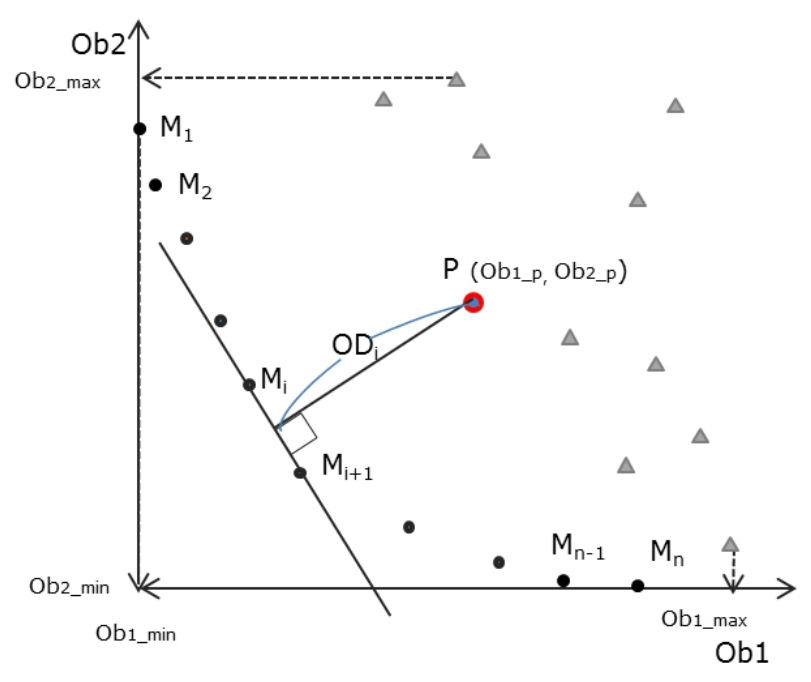

Fig. 7 Positions of original shape (P) and Pareto-front.

\section{Results}

\subsection{Results of optimization in the first stage}

Here, we describe the optimization process and results based on shape A called case A. In the same way, cases B, C, D, E, F, and G are optimizations based on shapes B, C, D, E, F, and G, respectively.

\subsubsection{Results of case $A$ in the first stage}

Figure 8 shows results of case $\mathrm{A}$ in the test function of $\mathrm{F} 1$, which used minimization of max WSS and minimization of surface area as the combination factors. Initial shapes for optimization with GA were generated on the basis of the original shape A (shown in Fig. 8(1)) with two control points randomly selected from the optimizing region (shown in Fig. 5(1)). The radius in the fixed region was set to the value of shape A (examples are shown in Fig. 5(2)). Those initial shapes were prepared excluding the original shape A. In the first stage, only radius of ICA was optimized. Center lines for two branches and the value of the radii of ECA were fixed to the values of shape A. Figure 8(2) shows the results of radii optimization of ICA. The vertical axis shows surface area, and the horizontal axis shows max WSS. The triangles are positions of shapes plotted with a value set of max WSS and surface area in the first generation, and the final Pareto-front shapes are plotted with blue dots. The search area of the early generation was scattered, but the Pareto-front was obtained in the final generation. Generally in the case of the two factors in a trade-off relationship, there are many solutions whose weight parameters for each factor are varied. Two examples on the Pareto-front are shown in Fig. 8(3): shape 1 (max time-averaged WSS: $2.78 \mathrm{~Pa}$, surface area: $0.00224 \mathrm{~m}^{2}$ ) has a higher weight on max WSS and a lower weight on surface area, and shape 2 (max time-averaged WSS: $10.13 \mathrm{~Pa}$, surface area: $0.00203 \mathrm{~m}^{2}$ ) has a higher weight on minimization of surface area and a lower weight on minimization of max WSS. Shape 2 is less suitable in terms of minimization of max WSS than shape 1, but it is an optimized shape with minimized surface area when max WSS is $10.13 \mathrm{~Pa}$.

Next, the position of shape A (max WSS: 2.92Pa, surface area: $0.00221 \mathrm{~m}^{2}$ ) was dotted at the graph, which was almost on the Pareto-front as shown in Fig. 8(2). Fig. 8(4) shows the radii of shape A and shape 3 (max WSS: 2.92Pa, 
surface area: $0.00210 \mathrm{~m}^{2}$ ), which was one of the shapes near to shape A on the graph among the optimized shapes. The average radii difference in optimization region was $1.3 \%$. There was only a small difference in radii between the two shapes.

We measured the degree of difference between optimized shapes and the original shape using ODI defined in the section 2.5. ODI, which was the difference in evaluated values of objectives, was $1.56 \times 10^{-4}$ (shown Table 1).
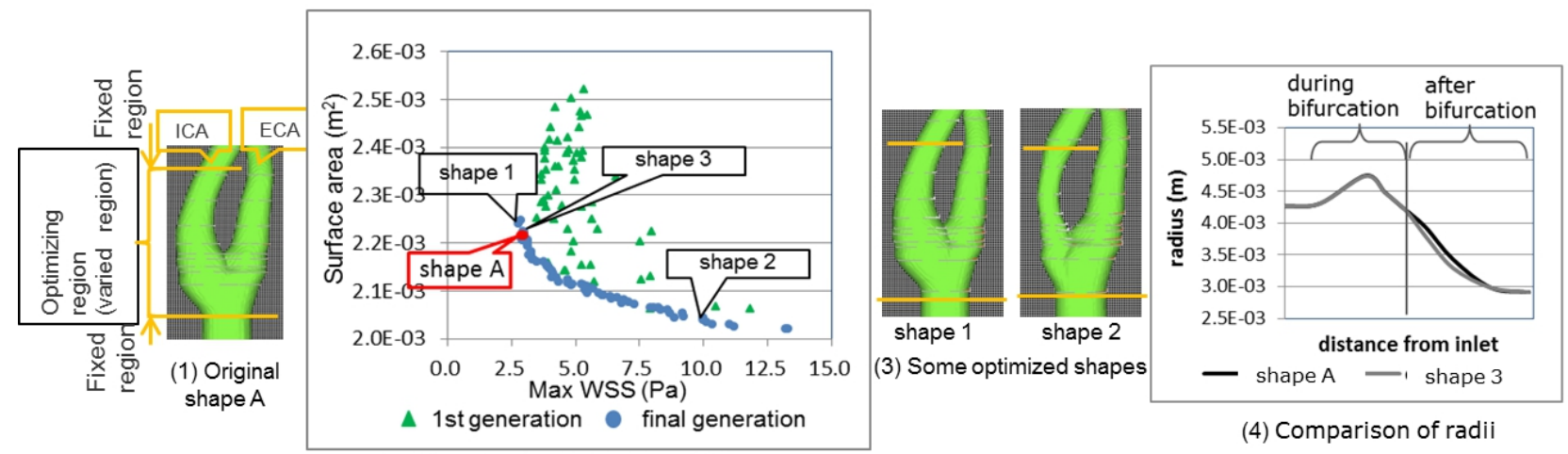

(2) Results of optimization of F1

(4) Comparison of radii

Fig. 8 Results of case A using F1 of the first stage. (1) Reconstructed original shape A. (2) Results of optimization of F1. Green triangles show the first generation of optimization, and blue dots show final Pareto-front shapes. The red dot is the position of shape A, which is on the Pareto-front. (3) Examples of optimized shapes. Shape 1 has higher weight to max WSS and lower weight to surface area, while shape 2 has higher weight to surface area and lower weight to max WSS (see panel a). (4) Comparison of radii of shape A and shape 3, which was one of the shapes near to shape A on the graph among the optimized shapes.

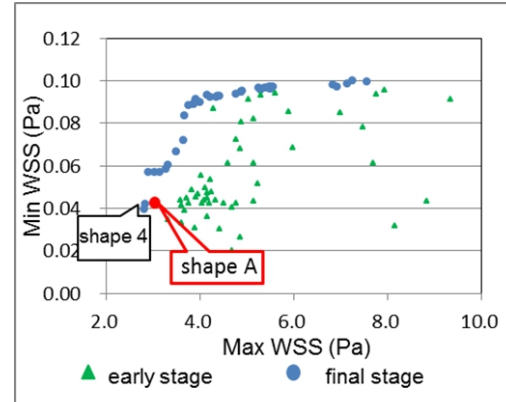

(1) Results of optimization of F2

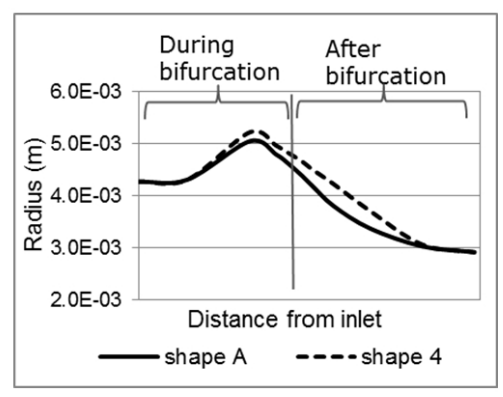

(2) Comparison of radii

Fig. 9 Results of case A using F2 in the first stage: F2 is the optimization of max WSS and maximization of min WSS as the objective function. (1) Results of optimization of F2. Blue dots are the positions of the final Pareto-front shapes. The red dot is the position of the original shape A that is not on the Pareto-front. (2) Comparison of radii of shape 4, which is the shapes nearest to shape A on the graph, and shape A.

Figure 9(1) shows results for F2 (minimization of max WSS and maximization of min WSS). In this case, ODI was 0.00845 , which was larger than that in the case of F1. The optimized shape nearest to shape A on the graph was shape 4 (max WSS: 2.82Pa, min WSS: 0.0422Pa), which had a smaller value of max WSS than shape A (max WSS: 2.92Pa, min WSS: $0.0426 \mathrm{~Pa}$ ). The radii of shape A differed from those of shape 4, which had larger radii overall than shape A (shown in Fig. 9(2)). The average radii difference between these shapes was $6.5 \%$. Shape A in the graph was not positioned on the Pareto-front, although it was near the front.

Fig. 10 shows the results from F3 to F6. Each black dot was each position of shape A. Shape 5 to 8 were the shapes nearest to shape A on the graph in the cases of F3 to F6, respectively. The radii of shape A differed from those of nearest ones. The average radii difference between shape A and each nearest shape (shape 5 to 8 ) was $8.7 \%$, 11.0\%, $6.1 \%$, and 5.4\%, respectively. ODIs of each test function are shown in Table 1. ODI in test function of F2 to F6 were larger than that in F1. Shape A was not positioned on any Pareto-front. 


\subsubsection{Results of cases $B, C, D, E, F$, and $G$ in the first stage}

Table 1 summarizes all tested functions from F1 to F6 in all cases. The right-most two rows show average values of each test function and its rank (in ascending order). The smallest ODI in test functions at each case is written in bold. In the six cases, ODI of F1 was the smallest, and that of F5 was the smallest in the only one case. The order of averaged value was $\mathrm{F} 1<\mathrm{F} 5<\mathrm{F} 2<\mathrm{F} 6<\mathrm{F} 3<\mathrm{F} 4$.

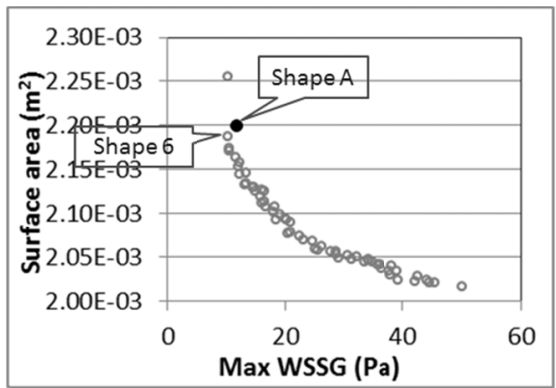

(1) Results of optimization of F3

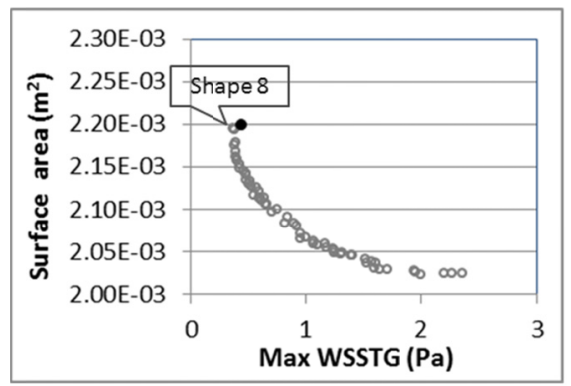

(3) Results of optimization of F5

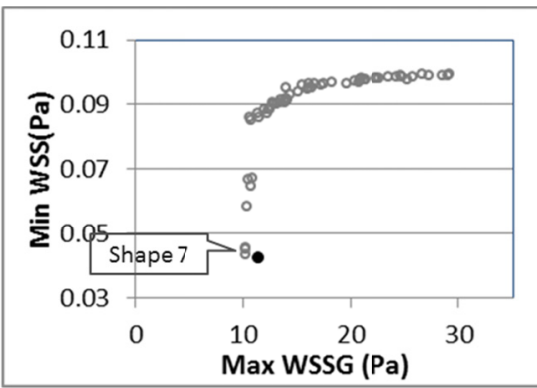

(2) Results of optimization of F4

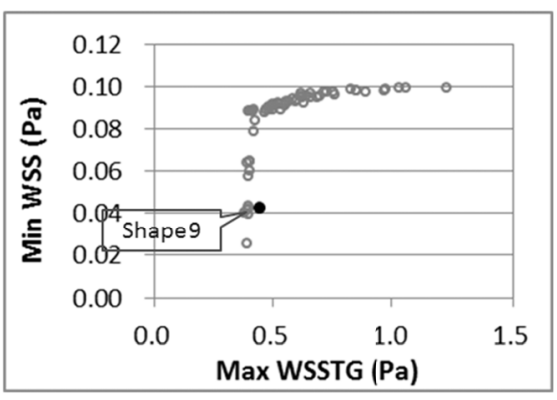

(4) Results of optimization of F6

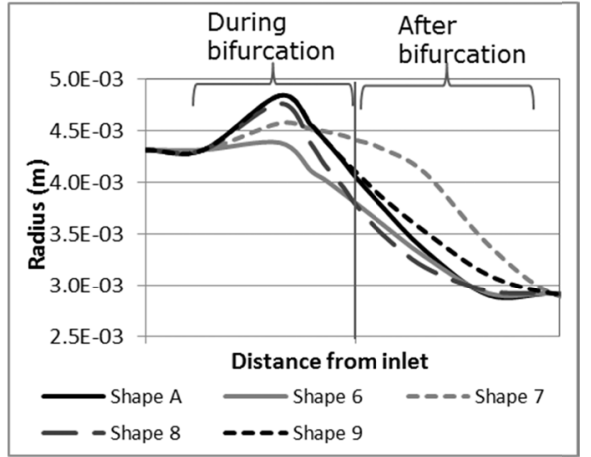

(5) Comparison of radi

Fig. 10 Results of case A of F3, F4, F5, and F6 in the first stage: (1) results of optimization of F3: minimization of max WSSG and minimization of inner surface area; (2) results of optimization of F4: minimization of max WSSG and maximization of min WSS; (3) results of optimization of F5: minimization of max WSSTG and minimization of inner surface area; and (4) results of optimization of F6: minimization of max WSSTG and maximization of min WSS. (5) Comparison of radii of shape 5 to 8 (which is the shapes nearest to shape A of each test function), and shape A.

Table 1 Summary of the results of optimization in the first stage The smallest ODI in each case is displayed in bold.

\begin{tabular}{|c|c|c|c|c|c|c|c|c|c|}
\hline $\begin{array}{l}\text { Test } \\
\text { function }\end{array}$ & Case A & Case B & Case C & Case D & Case E & \multirow{2}{*}{ Case F } & Case G & \multicolumn{2}{|c|}{ Summary } \\
\hline F1 & $\mathbf{1 . 5 6 \times 1 0}$ & $\mathbf{1 . 0 3 \times 1 0}$ & $\mathbf{4 . 6 2 \times 1 0}$ & $\mathbf{3 . 3 4 \times 1 0}$ & 0.00317 & $\mathbf{2 . 8 5 \times 1 0}$ & $\mathbf{0 . 0 0 2 1 5}$ & $9.51 \times 10^{-4}$ & 1 \\
\hline F2 & 0.00845 & 0.0262 & $8.34 \times 10^{-4}$ & 0.0716 & 0.00958 & 0.0107 & 0.02187 & 0.0213 & 3 \\
\hline F3 & 0.0245 & 0.00387 & 0.03013 & 0.0552 & 0.0357 & 0.0281 & 0.00565 & 0.0262 & 5 \\
\hline F4 & 0.0204 & 0.0485 & 0.02987 & 0.0516 & 0.0299 & 0.0287 & 0.0112 & 0.0315 & 6 \\
\hline F5 & 0.0215 & 0.0493 & 0.01424 & 0.0204 & $\mathbf{0 . 0 0 2 4 6}$ & 0.0170 & 0.00609 & 0.0187 & 2 \\
\hline F6 & 0.0135 & 0.0612 & 0.0209 & 0.0267 & 0.00328 & 0.0257 & 0.0126 & 0.0234 & 4 \\
\hline
\end{tabular}

\subsection{Results of optimization of the second stage}

As test F1 showed the best results at the first stage, we performed optimization using F1 by setting both the radius 
and center line of ICA as variables to study whether the factors (max WSS and surface area) were also related to the artery center line position or not. Those of ECA used the value of the original shape. In all seven cases, each original shape was on each Pareto-front. The results of case A were shown in Fig. 11, as an example. The black dot was the position of shape A. In all test cases (A to G), ODI was significantly small, the same as in the first stage (shown in Table 2).

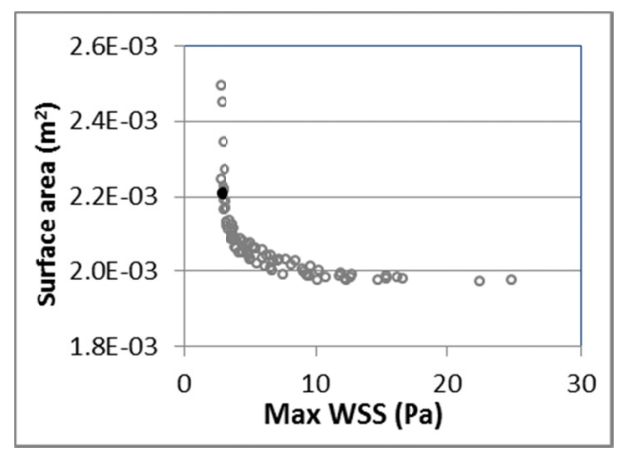

Fig. 11 Results in the second stage (case A of F1)

Table 2 Results of optimization of test function F1 in the second stage

\begin{tabular}{|c|c|c|c|c|c|c|c|c|}
\hline & Case A & Case B & Case C & Case D & Case E & Case F & Case G & $\begin{array}{c}\text { Summary } \\
\text { (Average) }\end{array}$ \\
\hline ODI & $1.06 \times 10^{-3}$ & $8.86 \times 10^{-4}$ & $2.95 \times 10^{-3}$ & $2.24 \times 10^{-4}$ & $1.56 \times 10^{-3}$ & $9.26 \times 10^{-4}$ & $1.62 \times 10^{-3}$ & $1.39 \times 10^{-3}$ \\
\hline
\end{tabular}

\section{Discussion}

Picking up five factors, we tested multi-objective optimization of six kinds of combination factors. In the results, optimized shapes were obtained in all test cases. Pareto-front was often partly spread sparsely because GA is a probability-based search method. One of them was F5 using case G (shown in Fig. 12). The original shape on the graph was positioned where there were a few points near it. We introduced ODI to validate whether the original is positioned on the Pareto-front or not even in such a case. ODI in F5 of case G was 0.00609 , which was a very small value (the second smallest value among six actual cases in F5).

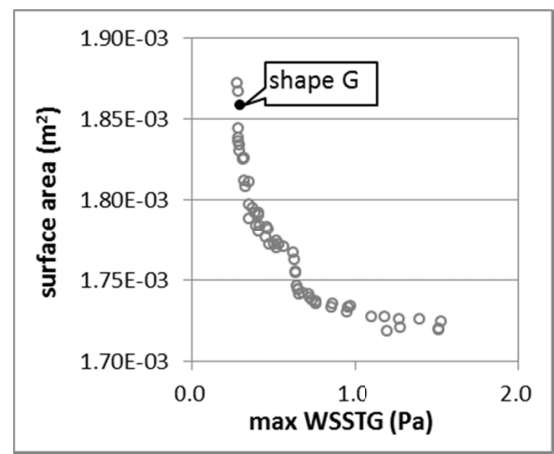

Fig. 12 Results of case G using F5 in the first stage: ODI was 0.00609.

In the results of the first stage (treating only radius as variable), ODI value of F1 was the smallest in the six cases, while F5 was the best in only one case. Average value of ODI of F1 was the smallest in six functions and about one-twentieth that of F5. It was indicated that the most relevant of the six tested combinations of factors was the combination of minimization both max WSS and inner surface area. These results show that the proposed method differentiated the relevance of selected factors.

Assuming that the artery shape is determined by locally sensible parameters, we chose WSS related factors which 
are sensed by endothelial cells (Chiu and Chien, 2011). The total surface is not locally sensible value but minimization of total surface area is possible to be regarded as a result of minimizing local diameter. In this study, the range of optimization region is only about three times of CCA diameter (at the position of the inlet), and the variation of radius we assumed in this region has smooth curve. Therefore, we surmise that local minimization of artery radius lead to minimum surface area in this calculation because the surface area is an integration of local circumference in the optimizing region.

Using ODI, we confirmed two factors (minimizing max time-averaged WSS and minimizing surface area) were effectively related to control radius. Many studies have suggested that hemodynamic factors are related to artery radius regulation, but no study has examined the relationship between hemodynamic factors and center line position. We found that those two factors are also effectively related to control the center line of artery.

This method could be effective to check other factors, not only hemodynamic factors but also physiological ones.

In the carotid bifurcation, there is carotid bulb which plays blood pressure control (Fadel, et al., 2009). There is a possibility that carotid bulb's biological function affects the bifurcation shape. However, as our results show the shape is affected with minimization of both max WSS and surface area, the biological need of carotid bulb would have less influence to the bifurcation shape. As these results only suggest relevance of factors but do not prove a causal relation between them, a fundamental experimental study or trace of artery regulation in the case of bypass surgery for example will be needed to validate them.

\section{Conclusion}

We proposed a new method to evaluate which hemodynamic factors are more relevant to artery shapes by using computer simulations with GA and CFD. Targeting carotid artery bifurcation, we tested six kinds of two-objective optimization functions in seven actual cases to study relevance of factors to artery radius. We measured the difference between the optimized shapes and the original shape with ODI, which was defined as the difference in the evaluated value of objectives. In the case of minimizing both max time-averaged WSS and inner surface area, ODI was the smallest (the best in six tested combinations). This method differentiated the relevance of selected factors. Moreover, it was revealed that this combination also relates to the center position of artery. Our method was confirmed to be very effective to study the relevance of uncertain factors.

\section{References}

Bao, X., Lu, C. and Frangos, J. A., Temporal gradient in shear but not steady shear stress induces PDGF-A and MCP-1 expression in endothelial cells, Arteriosclerosis Thrombosis Vascular Biology, (1999) No. 19, pp. 996-1003.

Bijari, P. B., Antiga, L., Gallo, D., Wasseman, B. A. and Steinman, D. A., Improved prediction of disturbed flow via hemodynamically-inspired geometric variables, Journal of Biomechanics, (2012) 45(9), pp. 1632-1637.

Canham, P. B., Finlay, H. M., Dixon, J. G., Boughner, D. R., and Chen, A. Measurements from light and polarised light microscopy of human coronary arteries fixed at distending pressure. Cardiovascular Research (1989) 23:973-982.

Chiu, J. J., Chen, L. J., Chen, C. N., Lee, P. L. and Lee, C. I., A model for studying the effect of shear stress on interactions between vascular endothelial cells and smooth muscle cells, Journal of Biomechanics, (2004) Vol. 37, pp. 531-539.

Chiu, J-J. and Chien, S., Effect of Disturbed flow on vascular endothelium: Pathophysiological basis and clinical perspectives, Physiological Reviews (2011) No. 91, pp. 327-387.

Dammers, R., Stifft, F., Tordoir, J. H., Hameleers, J. M., Hoe, A. P. s and Kreitner, P. J., Shear stress depends on vascular territory: comparison between common carotid and branchial artery, Journal of Applied Physiology (2003) 94, pp. 485-489.

Deb, K., Pratop, A., Agrawal, S. and Meyarivan, T., A fast elitist non-dominated sorting genetic algorithm for multi-objective optimization: NSGA2, IEEE Transactions on Evolutional Computation, (2002) 6-2, pp. $182-197$.

DePaola, N., Gimbrone, Jr, M., A., Davies, P. F. and Dewey, Jr, C. F.: Vascular endothelium responds to fluid shear stress gradients, Arteriosclerosis, Thrombosis, and Vascular Biology, (1993) Vol. 13, pp. 1254-1257.

Fadel, P. J., Ogoh, S., Keller, D. M. and Raven, P. B., Recent insights into carotid baroreflex function in humans using the variable pressure neck chamber, Experimental Physiology, (2009) 88-6, pp671-680.

Finlay, H. M., McCullough, L., and Canham, P. B., Three-dimensional collagen organization of human brain arteries at 
different transmural pressures, Journal of Vascular Research. (1995) 32, pp. 301-312.

Goldberg, D. E., Genetic algorithms in search, optimization, and machine learning, (1989) Addison Wesley, Reading, MA.

Hariton, I., deBotton, G. and Gasser, T. C., Stress-driven collagen fiber remodeling in arterial walls, Biomechanics and Modeling in Mechanobiology. (2007) 6, pp. 163-175.

Himeno, M. and Himeno, R., The niching method for obtaining global optima and local optima in multimodal functions, Systems and Computers in Japan, (2003) Vol. 34, pp. 30-42.

Himeno, M., Noda, S., Fukasaku, K. and Himeno, R., Parallel computation of GA search for the artery shape determinants with CFD, IOP Conference Series: Materials Science Engineering, (2010) Vol. 10. [Online] Available: iopscience.iop.org/1757-899X/10/1/012131.

Hoi, Y. Wasseman, B. A., Lakatta, E. G. and Steinman, D. A., Carotid bifurcation hemodynamics in older adults: effect of measured versus assumed flow waveform. Journal of Biomechanical Engineering, (2010) 132071006.

Jaimes, A. L. and Coello, C. A. C., MRMOGA: Parallel evolutionary multiobjective optimization using multiple resolutions, IEEE Congress on Evolutionary Computation, (2005) pp. 2294-2301.

Kamiya, A. and Togawa, T. Adaptive regulation of wall shear stress to flow change in the canine carotid artery, American Journal of Physiology, (1980) 239, pp. H14-H21.

Kelly, R. F. and Snow, H. M., Characteristics of the response of the iliac artery to wall shear stress in the anaesthetized pig, Journal of Physiology (2007) 582,2, pp. 731-743.

Langille, B. L. and O'Donnell, F., Reductions in arterial diameter produced by chronic decreases in blood flow are endothelium-dependent, Science, (1986) 231, pp. 405-407.

Masuda , H., Zhuang, Y-J., Singh , T. M., Kawamura, Murakami, K., M., Zarins, C. K. and Glagov, S., Adaptive remodeling of internal elastic lamina and endothelial lining during flow-induced arterial enlargement, Arteriosclerosis, Thrombosis, and Vascular Biology, (1999) 19, pp. 2298-2307.

Meng, H. Wng, Z., Hoi, Y. H., Goa, L., Metaxa, E., Swartz, D. D. and Kolega, J. K., Complex hemodynamics at the apex of an arterial bifurcation induces vascular remodeling resembling cerebral aneurysm initiation, Stroke, (2007) 38, pp. 1924-1931.

Noda, S., Fukasaku, K. and Himeno, R., Blood flow simulation using medical images without mesh generation, World Congress on Medical Physics and Biomedical Engineering, (2006) pp. 36-40.

Nowak, M., Structural optimization system based on trabecular bone surface adaptation, Structural Multidisciplinary Optimization, Vol. 32, (2006), pp. 241-249.

O’Fynn, P. M., O’Sullivan, G. and Pandit, A. S., Geometric variability of the abdominal aorta and its major peripheral branches, Annals of Biomedical Engineering, (2011) 38-3, pp. 824-840.

Ono, I. and Kobayashi, S., A real-coded genetic algorithm for function optimization using unimodal normal distribution crossover, Proceedings of $7^{\text {th }}$ International Conference on Genetic Algorithms, (1997) pp. 246-253.

Poepping, T. L., Nikolov, H. N., Rankin, R. N., Lee, M. and Holdsworth, D. W., An in vitro system for Doppler ultrasound flow studies in the stenosis carotid bifurcation, Ultrasound in Medicine \& Biology, (2002) No. 28(4), pp. 495-506.

Reneman, R. S., Arts, T. and Hoeks, A. P. G., Wall shear stress -an important determinant of endothelial cell function and structure - in the arterial system in vivo, Journal of Vascular Research, (2006) 43, pp. 251-269.

Sasaki, D., Yang, G. and Obayashi, S., Automated aerodynamic optimization system for SST wing-body configuration, Transaction of the Japan Society Aeronautical Space Science, (2004) Vol. 46, No. 154, pp. 230-237.

Schlz, U. G. R. and Rothwell, P. M., Major variation in carotid bifurcation anatomy, Stroke, (2001) 32, pp. $2522-2529$.

Sho, E., Nanjo, H., Sho, M., Kobayashi, M., Komatsu, M., Kawamura, K., Xu, C., Zarins, C. K. and Masuda, H. Arterial enlargement, tortuosity, and intimal thickening in response to sequential exposure to high and low wall shear stress, Journal of Vascular Surgery, (2004) Vol. 39-3, pp. 601-621.

Sorop, O., Spaan, J. A. E., Aweeney, T. E. and VanBavel, E., Effect of steady versus oscillating flow on porcine coronary arterioles. Involvement of NO and superoxide anion, Circulation Research, (2003) 22, pp. 1344-1351.

Thomas, J. B., Antiga, L., Che, S. L., Milner, J. S., Steinman, D. A. H., Spence, J. D., Rutt, B. K. and Steinman, D. A., Variation in the carotid bifurcation geometry of young versus older adults: Implications for geometric risk of atherosclerosis, Stroke, (2005) No. 36, pp. 2450-2456.

Takahashi, O. and Kobayashi, S.: An adaptive neighboring search using crossover-like mutation for multimodal 
function optimization, IEEE International Conference on System, Man and Cybernetics, (2001) pp. 261-267.

Tohda, K., Masuda, H., Kawamura, K., and Shozawa, T., Difference in dilatation between endothelium-preserved and desquamated segment in the flow-loaded rat common carotid artery, Arteriosclerosis, Thrombosis, and Vascular Biology, (1992) 2, pp. 519-528.

Tsubota, K., Adachi, T., and Tomita, Y., Functional Adaptation of Cancellous Bone in Human Proximal Femur Predicted by Trabecular Surface Remodeling Simulation toward Uniform Stress State, Journal of Biomechanics, Vol. 35-12, (2002), pp. 1541-1551.

\section{Appendix 1 How to reconstruct a three dimensional shape}

We used the same way in our previous work (Himeno et al., 2010). The essence of that is as follows. Figure13(1) is a diagram of cross-sectional shapes. $\mathrm{P}$ is the center line position, and $\mathrm{r}$ is the radius. From the CT or MRA medical images (shown in Fig.13(2)), before and after bifurcation, the cross-sectional shapes were approximated as a circle. During bifurcation, the cross-sectional shape of each branch in the regions facing each other was not approximated as a circle but was deformed with a spline function to fit to shapes of medical images. Outside of both branches, the shape is defined as a semicircle. Both branches were merged using spline interpolation. Figure 13(3) is the example of reconstructed cross-sectional shapes using this method. A three dimensional shape was reconstructed by smoothly connecting corresponding points on spline curves on each cross section.

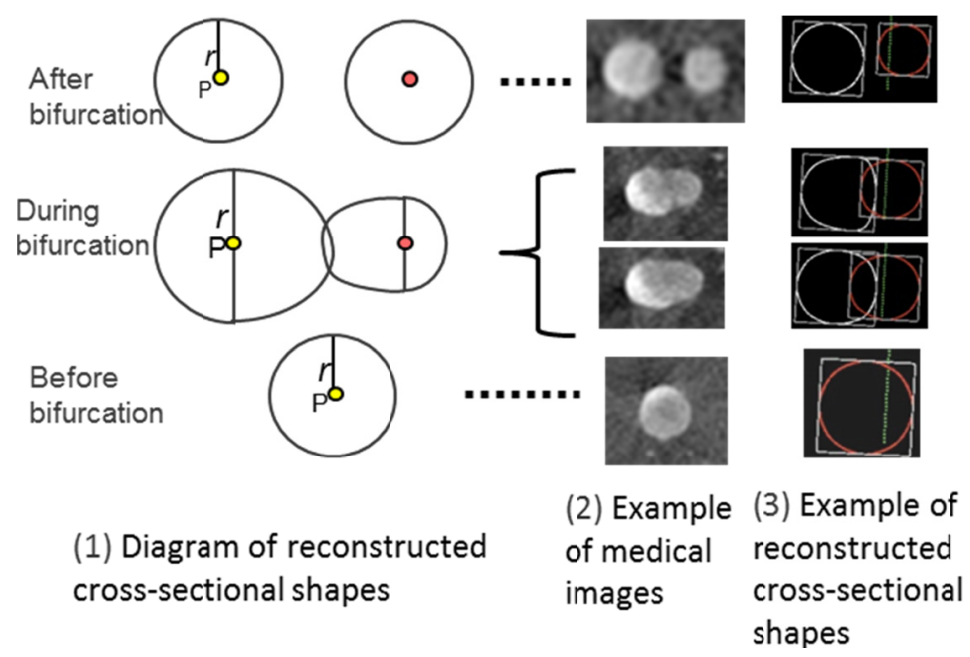

Fig. 13 How to reconstruct cross-sectional shapes.

\section{Appendix 2 Computation precision}

To check the error due to the voxel approach, we compared calculated results with theoretical Poiseuille flow $(\mathrm{Re}=1000)$ in a straight circular tube with a radius of 1 and a maximum flow rate of 1 . Figure 14 plots the error ratio for calculated WSS of three different voxel sizes: $0.2,0.1$, and 0.05 . The error ratio was defined as given Eq. 6 .

$$
\text { Error ratio }=\frac{\mid \text { theoretica l WSS }- \text { calcurated WSS } \mid}{\text { thoretical WSS }}
$$

Next, we checked if places where max or min of WSS related values stay same positions using all of seven original bifurcation shapes when calculating voxel size was changed. Figure 15 shows one of these results using shape A. In all cases, the changes are very small.

By using 32 CPUs (Intel Xeon X5570, 2.93GHz with InfiniBand X4 DDR), each blood flow simulation for voxel sizes of $0.2,0.1$, and 0.05 took 8 minutes, about 90 minutes, and about 32 hours, respectively. Considering computation precision and calculation time, we decided to use the voxel size of 0.1 for blood flow simulations. 


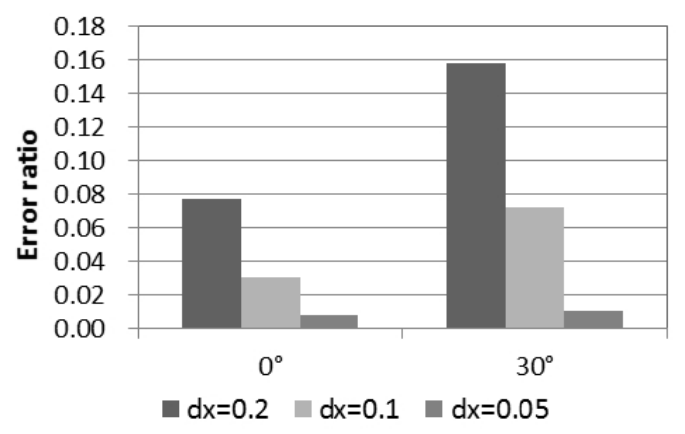

Fig. 14 Error ratios for calculated WSS (see equation (6)) of three different voxel sizes: $0.2,0.1$, and 0.05 .

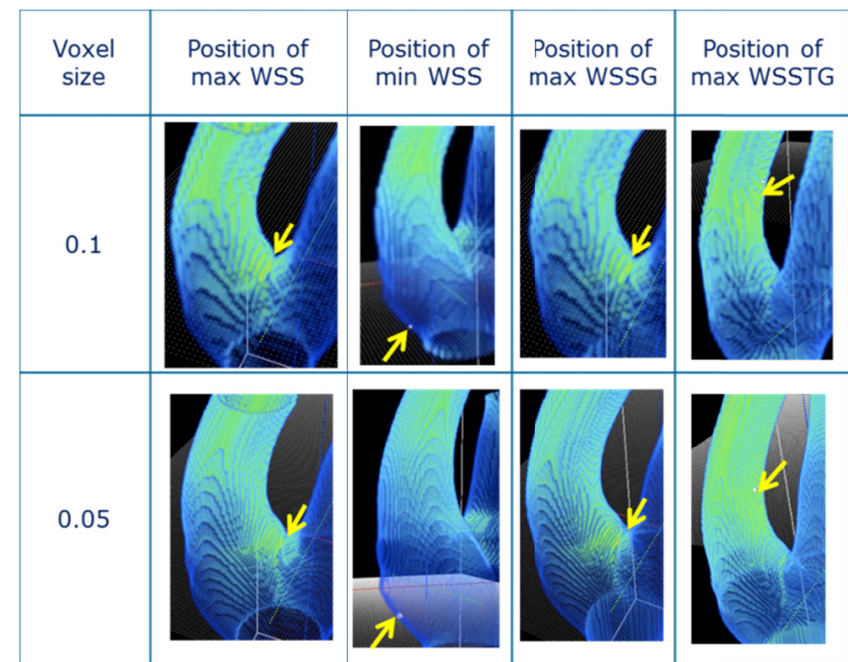

Fig. 15 WSS, WSSG, and WSSTG distribution for voxel sizes of 0.1 and 0.05 . Yellow arrows show the positions of each point.

\section{Appendix 3 How to solve multi-objective optimization problem}

Some of the problems were multi-objective optimization, which involves trade-offs between objective functions. Figure 16 shows a scheme of an optimization graph in the case of minimization of two objectives (Objects 1 and 2). The grey part shows the feasible region for solutions (search domain). When objectives have a trade-off relationship, no single solution satisfies all objectives, but many solutions have varied weight parameters for each objective (bold curve in Fig. 16), which are called the Pareto-front. The goal of multi-objective optimization is to find this Pareto-front, a set of solutions (green circles in Fig. 16) on this curve.

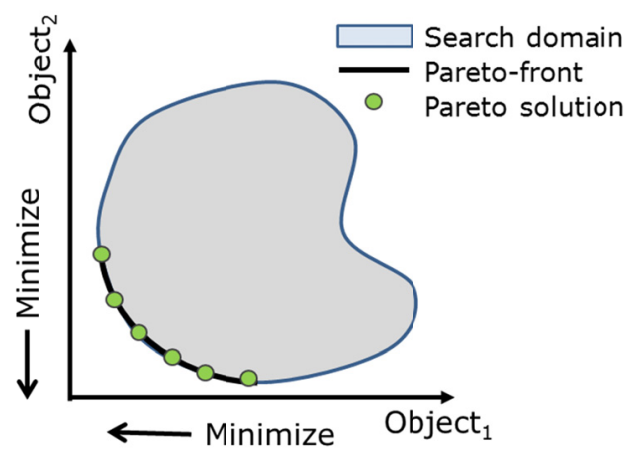

Fig. 16 Pareto-front in the case of minimization of two objectives (Object1 and 2). Bold curve is Pareto-front, which is solution of multi-objective optimization.

We used the Multi-Objective Genetic Algorithm (MOGA) for shape optimization. Figure 17 shows the outline of MOGA. First, candidate shapes (blue circles) are generated randomly as the initial population (initial generation). Second, new shapes (yellow circles) are generated referring to some shapes near to the Pareto-front (operation (a)). Third, solutions nearer to the Pareto-front are selected as the candidate solutions for next generation (operation (b)). The change from (a) to (b) is called a generation. The Pareto solutions (green circles) are found after several generations. Shapes are generated and selected probabilistically, which enables solutions to be searched for globally and prevents solutions being restricted to only local ones. 


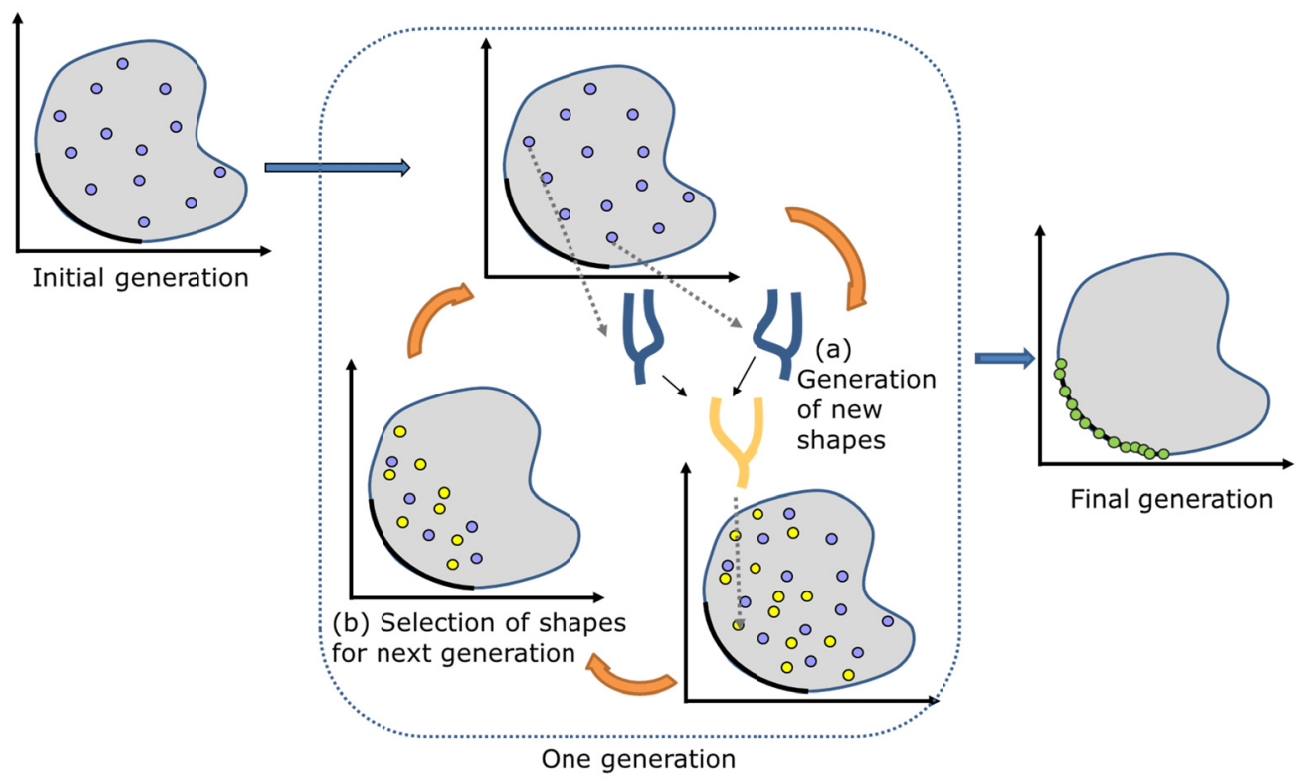

Fig. 17 Outline of MOGA. Pareto solutions (green circles) are searched for by repeating change (a) to (b) using initial shapes (blue circles). 